\title{
A 3D view of the planetary nebula NGC 40
}

\section{Hektor Monteiro and Diego Falceta-Gonçalves}

\author{
Departamento de Física e Química, Universidade Federal de Itajubá \\ email: hektor.monteiro@gmail.com
}

\begin{abstract}
We present the results of a study of the planetary nebula NGC 40 with the use of the 3-D photoionization code Mocassin constrained by observational data of different types. The modeling process allows us to derive the three-dimensional nebular structure, physical and chemical characteristics and ionizing star parameters of the object by simultaneously fitting the integrated line intensities, the temperature map, density map, and the observed morphologies in different emission lines. For this particular case we combined hydrodynamical simulations with the photoionization scheme in order to obtain a self-consistent distribution of density and velocity of the nebular material. Finally, using theoretical evolutionary tracks of intermediate and low mass stars, we estimate the mass and age of the central star of NGC 40 as being $0.57 \mathrm{M}_{\odot}$ and $5810 \mathrm{yr}$, respectively. The distance obtained from the fitting procedure was $1150 \pm 170 \mathrm{pc}$.
\end{abstract}

Keywords. planetary nebulae: individual (NGC 40), methods: numerical

\section{Introduction}

Planetary nebulae are end products of the evolution of stars with masses below $8 M_{\odot}$ and as such have great importance in many fields in astrophysics, from basic atomic processes in solar like stars to distant galaxies. Although the general picture of planetary nebulae formation is well understood (Kwok 2008) many questions remain unsolved, such as the mechanism by which the stellar ejecta end up forming the many observed morphologies.

In the past $\mathrm{PNe}$ have been studied with empirical methods and one-dimensional photoionization models. This scenario has recently been significantly changed with modern computational capabilities. As we have discussed in the previous papers of this series Monteiro et al. (2004), Monteiro et al. (2005) and Schwarz \& Monteiro (2006), precise distances are of paramount importance to the study of these objects. To this extent our work provides precise, self-consistently determined distances for objects with comprehensive, in most cases spatially resolved, observational constraints. These objects can provide valuable calibration to pre-existing distance scales as well as self-consistently determined physical and chemical quantities.

\section{Results}

The results of the modeling process show good agreement with all observables chosen as constraints to the modeling. The usual diagnostic maps (density, temperature) as well as all emission line maps obtained by Leal-Ferreira et al. (2010) are nicely reproduced. We also reproduce with a good degree of agreement the kinematical data obtained by Sabbadin et al. (2000).

The abundances obtained are shown in Table 1. By comparing the values of effective temperature and luminosity obtained from our model (see Fig. 1), $T_{\text {eff }}=(50 \pm 5) \mathrm{kK}$ and $L=(1719 \pm 170) \mathrm{L}_{\odot}$, to evolutionary tracks of Vassiliadis \& Wood (1994) we estimate an age of $(5810 \pm 600) \mathrm{yr}$, for a core mass of $(0.57 \pm 0.06) \mathrm{M}_{\odot}$ formed from a progenitor with $(1 \pm 0.1) \mathrm{M}_{\odot}$. The age value is in good agreement with literature values. 
Table 1. Comparison of literature abundance determinations for NGC 40.

\begin{tabular}{lccccc}
\hline work & $\mathrm{He} / \mathrm{H}$ & $\mathrm{O} / \mathrm{H}$ & $\mathrm{N} / \mathrm{H}$ & $\mathrm{S} / \mathrm{H}$ & $\mathrm{C} / \mathrm{H}$ \\
\hline Perinotto et al. 2004 & $4.73 \mathrm{e}-2$ & $7.24 \mathrm{e}-4$ & $8.86 \mathrm{e}-5$ & $2.80 \mathrm{e}-6$ & - \\
Liu et al. 2004 & $1.2 \mathrm{e}-1$ & $4.9 \mathrm{e}-4$ & $8.5 \mathrm{e}-5$ & $2.6 \mathrm{e}-6$ & $6.9 \mathrm{e}-4$ \\
Clegg et al. 1983 & $>0.044$ & $8.4 \mathrm{e}-4$ & $2.4 \mathrm{e}-4$ & $3.9 \mathrm{e}-6$ & $1.0 \mathrm{e}-3$ \\
Pottasch \& Bernard-Salas & $>0.046$ & $5.3 \mathrm{e}-4$ & $1.3 \mathrm{e}-4$ & $5.6 \mathrm{e}-6$ & $1.9 \mathrm{e}-3$ \\
This work & $0.9 \mathrm{e}-1$ & $1.9 \mathrm{e}-4$ & $6.8 \mathrm{e}-5$ & $6.8 \mathrm{e}-6$ & $6.5 \mathrm{e}-4$ \\
\hline
\end{tabular}

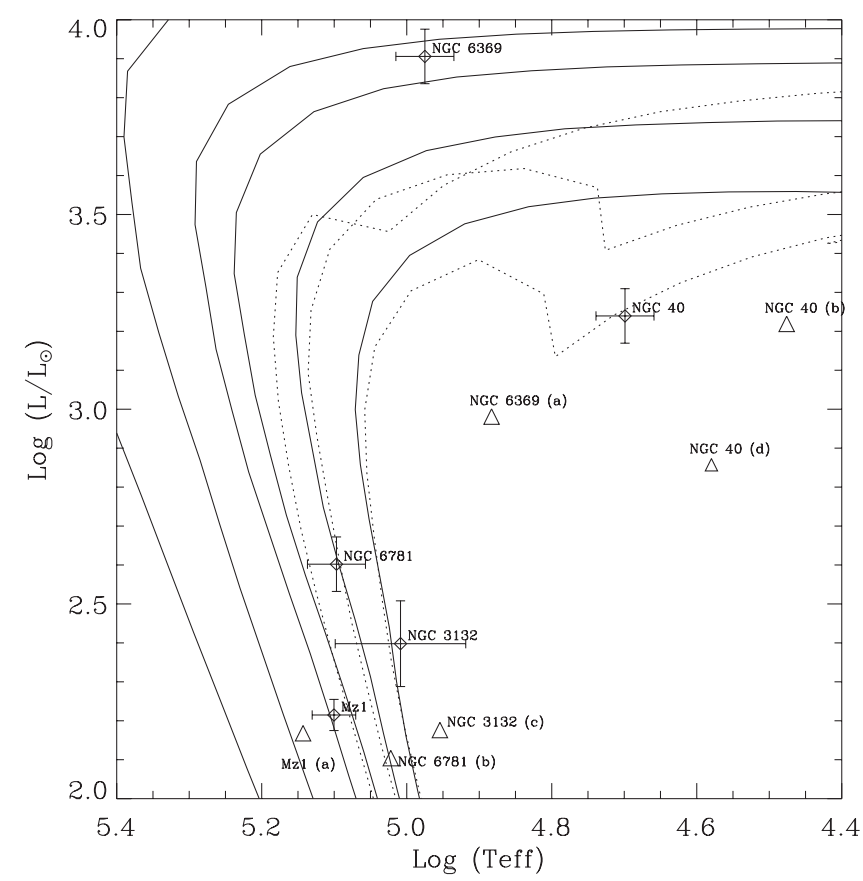

Figure 1. HR diagram for all PNe that had their central star properties determined by our method. Also plotted are the literature values for comparison (for details see Monteiro \& Falceta-Gonçalves 2011).

\section{Acknowledgements}

The author would like to thank CNPq grant 573648/2008-5 and the IAU Grants for financial support.

\section{References}

Clegg, R. E. S., Seaton, M. J., Peimbert, M., \& Torres-Peimbert, S. 1983, MNRAS, 205, 417

Kwok, S. 2008, IAU Symposium No. 252, 197

Leal-Ferreira, M. L., Gonçalves, D. R., Monteiro, H., \& Richards, J. W. 2010, MNRAS, 1759

Liu, Y., Liu, X.-W., Luo, S.-G., \& Barlow, M. J. 2004, MNRAS, 353, 1231

Monteiro, H., \& Falceta-Gonçalves, D. 2011,ApJ,738, 174

Monteiro, H., Schwarz, H. E., \& Gruenwald, R., Heathcote,S. R. 2004, ApJ, 609, 194

Monteiro, H., Schwarz, H. E., Gruenwald, R., \& Guenthner, K., Heathcote,S. R. 2005, ApJ, 620, 321

Perinotto, M., Morbidelli, L., \& Scatarzi, A. 2004, MNRAS, 349, 793

Pottasch, S. R. \& Bernard-Salas, J. 2010, A\&A, 517, A95

Sabbadin, F., Cappellaro, E., Benetti, S., Turatto, M., \& Zanin, C. 2000, A\& A, 355, 688

Schwarz, H. E. \& Monteiro, H. 2006, ApJ, 648, 430

Vassiliadis, E. \& Wood, P. R. 1994, ApJS, 92, 125 